patients, no change in $20.8 \%$, and symptoms were worse in $8.3 \%$. Compared to the previous 6 months, seizures were increased in number in 2 children $(8.3 \%)(\mathrm{P}<0.001)$, and not increased in $22(91.6 \%) ; 7$ patients had fewer seizures; $58.3 \%$ had partial epilepsy and $41.7 \%$ generalized epilepsy. ADHD was inattentive type in $41.7 \%$, combined type in $37.5 \%$, and $20.8 \%$ hyperactive/impulsive type. Results of 11 previous reports of MPH, ADHD and epilepsy are summarized: 5 studies reported an increase in seizures and 6 showed no increase. One controlled study of OROS-MPH observed a daily increased risk of seizures with increasing doses of the stimulant. (Koneski JAS, Casella EB, Agertt F, Ferreira MG. Efficacy and safety of methylphenidate in treating ADHD symptoms in children and adolescents with uncontrolled seizures: A Brazillian sample study and literature review. Epilepsy Behav July 2011;21:228-232). (Respond: Dr Julio AS Koneski. E-mail: juliokoneski@neurologica.com.br).

COMMENT. The majority of studies reporting no or small risk of seizures with MPH and epilepsy involve patients whose seizures are controlled with AEDs. Few studies investigate the safety of MPH and other stimulants in treatment of ADHD in children with epilepsy or epileptiform EEG, not treated with AEDs.

In a group of 234 children with uncomplicated ADHD who received EEGs, 36 (15.4\%) showed epileptiform abnormalities, $40 \%$ rolandic spikes and $60 \%$ focal abnormalities. With introduction of stimulant therapy in $205(87.6 \%)$ patients, seizures occurred only in the stimulant-treated group: in one of $175(0.6 \%)$ patients with a normal EEG and in $3(10 \%)$ of 30 with epileptiform EEGs. Seizures occurred in 2 of $12(16.7 \%)$ with EEG rolandic spikes (Hemmer SA, Pasternak JF et al. Pediatr Neurol 2001;24(2):99-102). The authors suggest that an epileptiform EEG in neurologically normal children with ADHD predicts considerable risk for the occurrence of seizures during stimulant therapy whereas a normal EEG carries minimal and no increased risk.

\title{
COMPUTER-ASSISTED MANAGEMENT OF ADHD
}

Researchers at the Departments of Psychiatry and Pediatrics, Children's Memorial Hospital, Chicago, conducted a randomized medication trial for ADHD involving 24 pediatric practices. Patients were randomly assigned to 2 groups, treatment as usual or a specialized care group in which physicians received 2 hours of ADHD medication management training plus training on a software program to monitor response. Parent and teacher reports were obtained before treatment and 4, 9, and 12 months after starting medication. Exclusion criteria included IQ $<70$, history of intolerance to stimulants, and ADHD medication in previous 2 months. Of 270 children participating, 208 (77\%) were boys and $62(23 \%)$ girls, mean age 8.2 years. Comparisons between groups were made for the highest daily dose of medication, with stimulant medications converted to methylphenidate equivalents. Total daily medication dose did not differ significantly between groups: mean $27.1 \mathrm{mg} /$ day for titration group and $27.9 \mathrm{mg} /$ day for patients not adhering to dose titration. Children in both groups improved on the ADHD Rating Scales and SNAP-IV, with no group differences in rate of improvement. Starting on a shortacting versus long-acting stimulant showed no significant difference in benefit but significant effects were associated with progressing sequentially until symptoms were in the average range $(\mathrm{P}=0.039)$. Brief physician training alone was of no added benefit, but 
adherence to recommended titration procedures was followed by improvements in total and inattentive ADHD symptoms on the ADHD Rating Scales and SNAP-IV parent and teacher scales. Computer-assisted medication management may contribute to better treatment outcomes in pediatric primary care management of ADHD. (Lavigne JV, Dulcan MK, LeBailly SA, Binns HJ, Cummins TK, Jha P. Computer-assisted management of attention-deficit/hyperactivity disorder. Pediatrics July 2011;128(1):e46e53). (Respond: John V Lavigne PhD, Department of Child and Adolescent Psychiatry (\#10), Children's Memorial Hospital, 2300 Children's Plaza, Chicago, IL 60614. E-mail: jlavigne@childrensmemorial.org).

COMMENT. Successful management of the child with ADHD is benefitted by computer-assisted titration of medications. Brief training periods for physicians are not sufficient to obtain a better outcome. Computer-assisted dose-response decision-making increases adherence to guidelines. The optimal dose is the lowest dose producing a clinically significant change and symptom rating in the average range. This is associated with significant improvements in classroom behavior. Practices that stress to the parents the importance of obtaining school reports are more likely to obtain optimal results.

\section{NEUROMUSCULAR DISORDERS}

\section{COURSE OF SELENOPROTEIN-RELATED MYOPATHIES}

The clinical course and genotype-phenotype correlations in 41 patients aged 1-60 years with selenoprotein-related myopathy (SEPNRM) due to SEPN1 gene mutations were evaluated retrospectively in a study at The Dubowitz Neuromuscular Center, London, and other centers in the UK. Mean age at onset was 2.7 years, ranging from birth to second decade. One third were congenital in onset with hypotonia. In $46 \%$, onset was at 6 months to 5 years of age with delayed motor milestones. Creatine kinase was minimally elevated in $16 \%$ and markedly elevated only in 1 . All but 2 patients remained independently ambulant. Respiratory insufficiency generally developed and nocturnal noninvasive ventilation was started at a mean age of 13.9 years. Scoliosis preceded by rigid spine developed at a mean age of 10 years and was treated surgically at 13.9 years. Joint contractures were present in 26 (63\%) at a mean age of 10.4 years; 2 fatients had finger contractures at birth. Motor abilities remained static over time. Twenty of 35 $(57 \%)$ were underweight. Two patients died from respiratory failure at age 10 and 22 years. Muscle biopsy performed in 34 patients showed multiminicores and nonspecific myopathic changes. Genetic sequencing showed 14 new SEPNI mutations, 48\% missense. (Scoto M, Cirak S, Mein R, et al. SEPN1-related myopathies. Clinical course in a large cohort of patients. Neurology June 14, 2011;76(24):2073-2078). (Respond and reprints: Dr Francesco Muntoni, The Dubowitz Neuromuscular Centre, UCL Institute of Child Health, 30 Guilford Street, London WCIN 1EH, UK. E-mail: f.muntoni@ich.ucl.ac.uk).

COMMENT. This study expands the spectrum of myopathy with SEPNI mutations, with respect to severity of the disease, age at onset, and long-term outcome. 